\title{
Remarkable similarity of force induced dsRNA conformational changes with stretched dsDNA and their detection using electrical measurements : Supplementary Information
}

\author{
Abhishek Aggarwal, ${ }^{1}$ Saientan Bag, ${ }^{1}$ and Prabal K. Maiti ${ }^{1,}$, \\ ${ }^{1}$ Center for Condensed Matter Theory, Department of Physics, \\ Indian Institute of Science, Bangalore-560012, India
}

(Dated: August 31, 2018)

\section{HOPPING MODEL USED FOR CHARGE TRANSPORT CALCULATIONS}

In our model, we use nearest neighbour hopping, in which, a charge present at any hopping site other than terminal bases will have 5 destinations to hop to, i.e. 4 hopping sites from two adjacent base pairs and $5^{\text {th }}$ hopping site will be the complementary base of the present site, as shown in fig $\mathrm{S} 1$.

$\Delta G_{i j}^{e x t}$ is the contribution due to the external electric field, taken as potential difference between two sites in our calculations. We take uniform distribution of potential between the base pairs, i.e. consecutive base pairs will have a potential difference of $\left(\frac{V}{(N-1)}\right)$ (fig. S1, while bases of same pairs will have zero potential difference i.e.

$\Delta V=\left\{\begin{array}{l}\left(\frac{V}{N-1}\right), \text { for consecutive bases along the helical axis of DNA or RNA along positive voltage. } \\ 0, \text { for base pairs at the same level along the helical axis. } \\ -\left(\frac{V}{N-1}\right), \text { for consecutive bases along the helical axis of DNA or RNA along negative voltage. }\end{array}\right.$

Whereas,

$$
\Delta G_{i j}^{i n t}=U_{i}^{c C}-U_{i}^{n N}+U_{j}^{c C}-U_{j}^{n N}
$$

Where, $U_{i}^{n N}\left(U_{i}^{c C}\right)$ is the internal energy of neutral (charged) base in neutral (charged) state geometry.

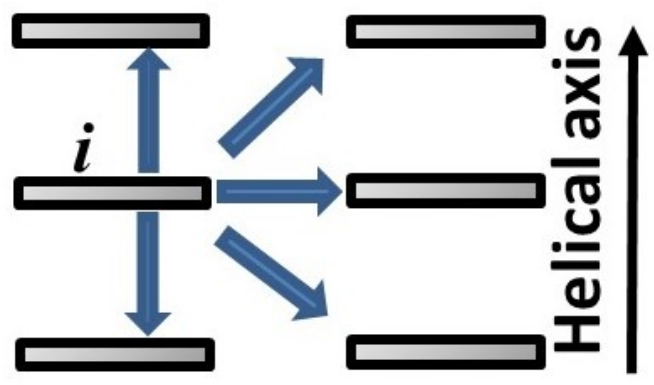

(a)



(b)

FIG. S1: (a) Schematic diagram describing the available hopping sites (i.e. the nucleobases) for a charge present at a charge hopping site $i$. A charge present at a site other than terminal bases will have 5 sites to hop to. (b) Distribution of voltage along the dsDNA or dsRNA chain during charge transport calculations. Here, we have assumed that the potential is distributed uniformly along the RNA chain. 


\section{SNAPSHOTS FOR SLOW PULLING RATE}

In the main text, snapshots for all the pulling protocols are shown for a pulling rate of $0.0001 \mathrm{pN} / \mathrm{fs}$. Here, snapshots for OS3 and OS5 pulling protocols are shown for a 10 times slower pulling rate simulation, i.e. $0.00001 \mathrm{pN} / \mathrm{fs}$. Fig. S3 shows that for OS5 case, the Force Induced Melted (FIM) state is achieved at around $100 \mathrm{pN}$, which is less than compared to that of higher pulling rate case presented in the main text. Similarly in OS3 case (fig. S22, traits of S-RNA are seen around $200 \mathrm{pN}$ which is again less than the $0.0001 \mathrm{pN} / \mathrm{fs}$ case value. However, both OS3 and OS5 cases show similar trends for all the pulling rates used. This shows that pulling rate affects the results at a quantitative level only.

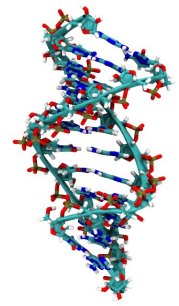

(a) $0 \mathrm{pN}$

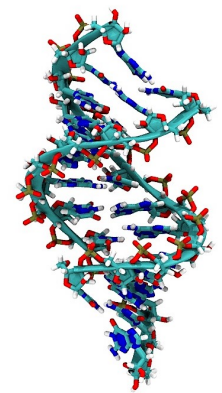

(b) $50 \mathrm{pN}$



(c) $100 \mathrm{pN}$

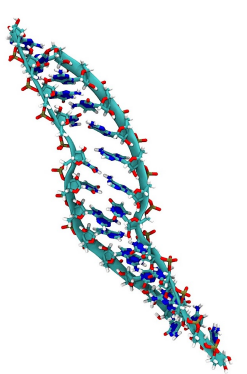

(d) $150 \mathrm{pN}$

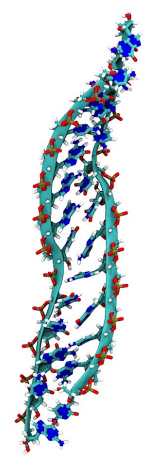

(e) $200 \mathrm{pN}$

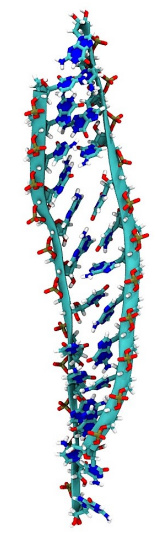

(f) $250 \mathrm{pN}$

FIG. S2: Instantaneous snapshots of OS3 pulling protocol for a pulling rate of $0.00001 \mathrm{pN} / \mathrm{fs}$ at various forces. The traits of S-RNA can be seen from $150 \mathrm{pN}$ onwards.

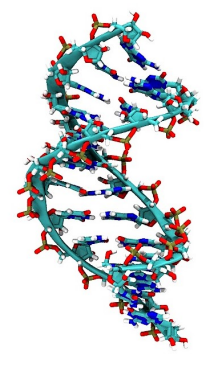

(a) $0 \mathrm{pN}$



(b) $50 \mathrm{pN}$

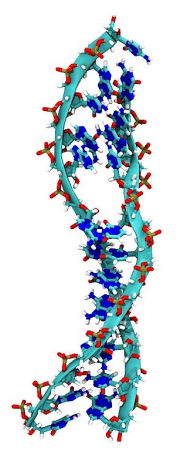

(c) $100 \mathrm{pN}$

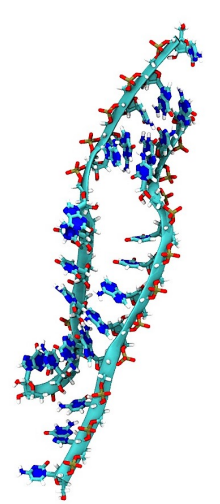

(d) $150 \mathrm{pN}$

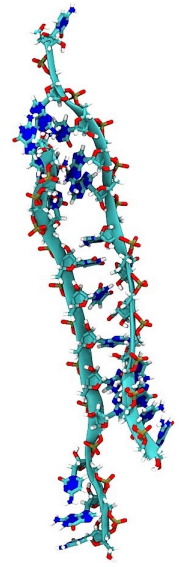

(e) $200 \mathrm{pN}$

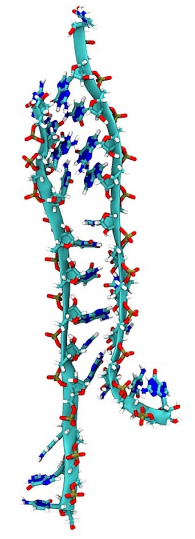

(f) $250 \mathrm{pN}$

FIG. S3: Instantaneous snapshots of OS5 pulling protocol for a pulling rate of $0.00001 \mathrm{pN} / \mathrm{fs}$ at various forces. Clearly, dsRNA melts around $150 \mathrm{pN}$. 


\section{DISTRIBUTION OF LUMO ORBITALS}

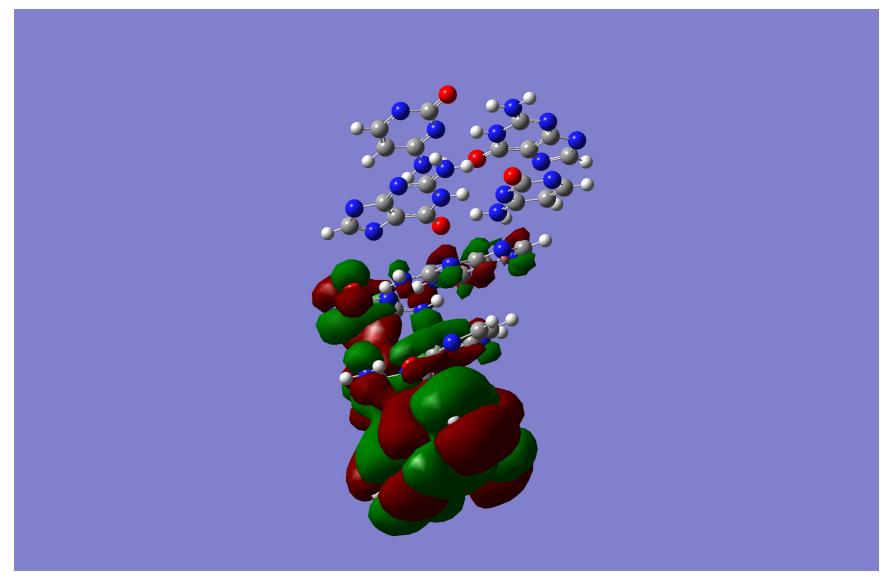

(a)

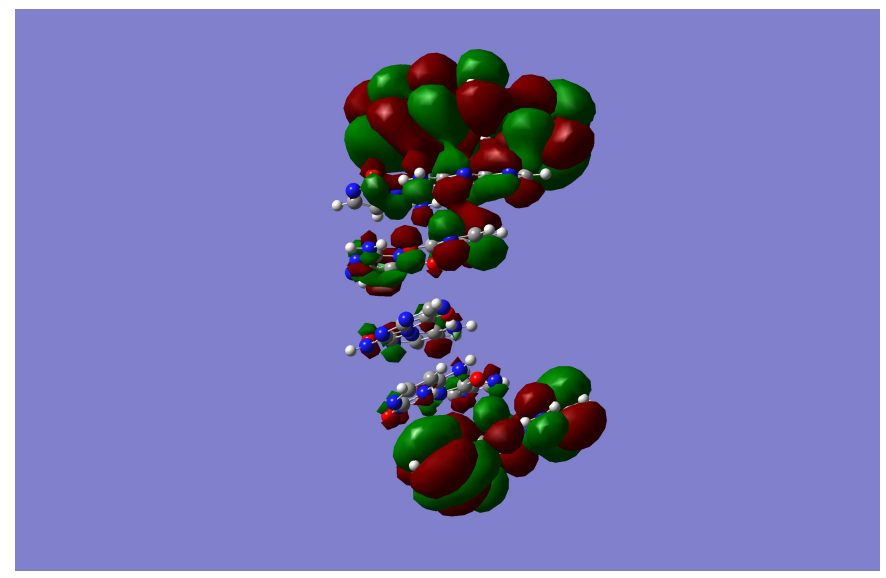

(c)

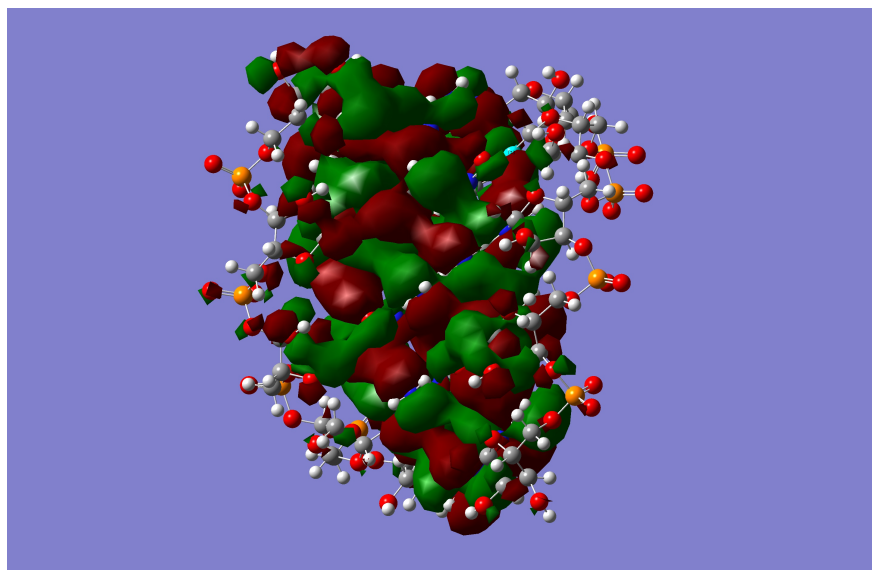

(b)

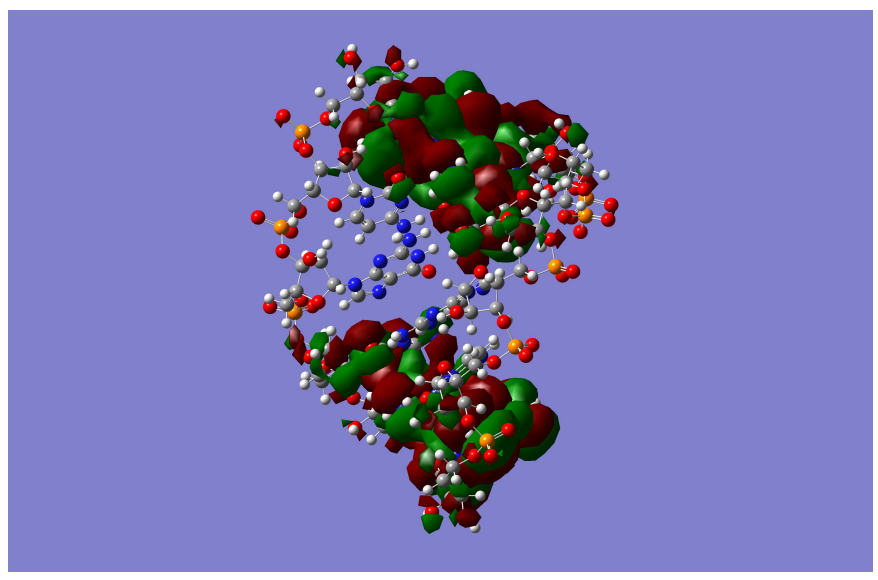

(d)

FIG. S4: Distribution of LUMO orbitals on 5 bp (d-(CGCGC)) and 6 bp long dsRNA sequence (d-(CGCGCG)) for a isovalue of 0.002 (a) without backbone and (b) with backbone included. As the LUMO orbitals are not distributed on the backbone atoms, the backbone atoms are neglected during the current calculations.

Fig. S4 shows the distribution of LUMO orbitals along the dsRNA chain with and without backbone for 5 and 6 base pair (bp) long dsRNA sequence. The LUMO orbitals for both the cases are not distributed on the backbone atoms of dsRNA. This supports the fact that backbone atoms do not take part in charge transport. 


\section{COMPARISON OF EFFECT OF USING DIFFERENT FORCE FIELDS}

Simulations were also carried out using two other force fields: (1) combination of parmbsc0[1] and Yildirim [2] $\chi$ modifications to ff99 and (2) a recently developed force field by Aytenfisu et al. [3]. Equilibrium simulations were carried out for 200ns for these two force fields. A comparison of helical parameters shows (fig. S5) that RNA maintains its structure during all the three cases.

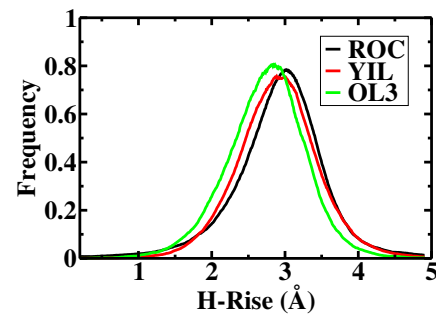

(a)

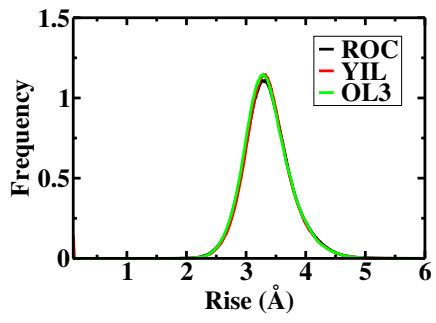

(b)

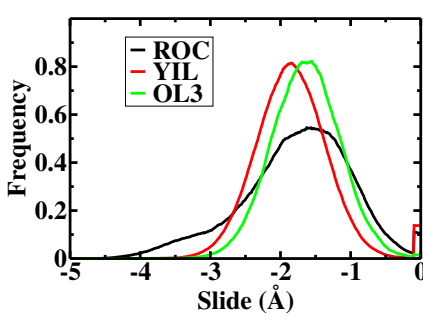

(c)

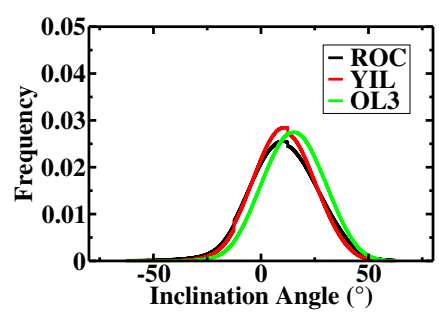

(d)

FIG. S5: Structural parameters (a) Helical-rise, (b) Rise, (c) Slide and (d) Inclination for different force fields used. All the structural parameters are similar for all three force fields.

We also performed non-equilibrium pulling simulations using the two force fields for OS3 and OS5 protocols using two different pulling rates: $0.0001 \mathrm{pN} / \mathrm{fs}$ and $0.00001 \mathrm{pN} / \mathrm{fs}$. Figure $\mathrm{S} 6$ shows the variation of number of H-bonds as the force is increased at a pulling rate of $0.0001 \mathrm{pN} / \mathrm{fs}$. In all the cases, more number of $\mathrm{H}$-bonds remains intact as a function of force for OS3 case in comparison to OS5 case, indicating the emergence of S-RNA in OS3 case. For YIL force field, there is an initial dip in the number of H-bonds, but as a function of time it increases and eventually maintains its structure in S-RNA form.

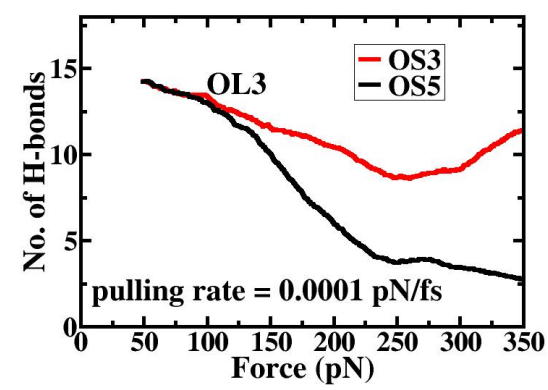

(a)

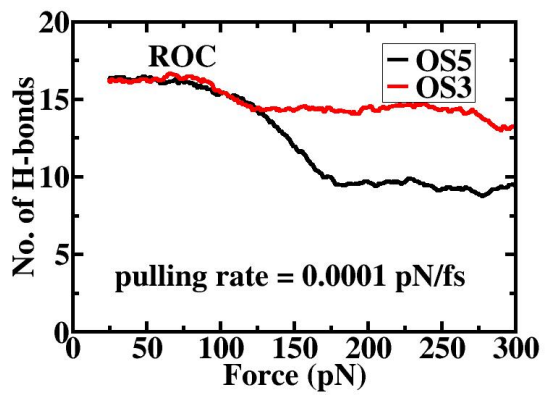

(b)

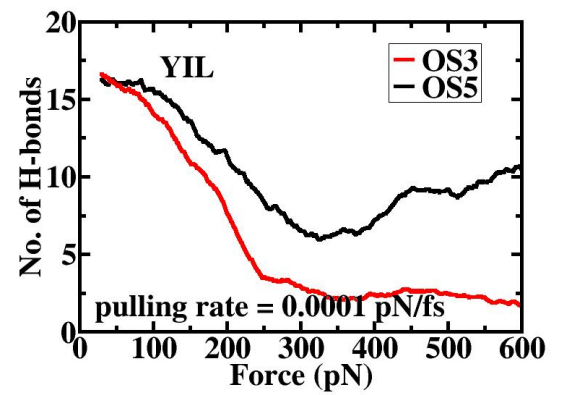

(c)

FIG. S6: Number of H-bonds vs applied force curves for different force fields at a pulling rate of $0.0001 \mathrm{pN} / \mathrm{fs}$. It is evident that for OS3 protocol, more number of H-bonds are maintained in OS3 protocol than OS5 protocol in all the force fields.

Figure S7 shows the variation of number of H-bonds as the force is increased at a pulling rate of $0.00001 \mathrm{pN} / \mathrm{fs}$. Here as well, more number of H-bonds is maintained for OS3 case and S-RNA is seen at lower range of forces as seen in the case when OL3 FF was used. This analysis shows that the emergence of S-RNA in OS3 protocol is quite robust and reproduced using three different well known RNA FF. 


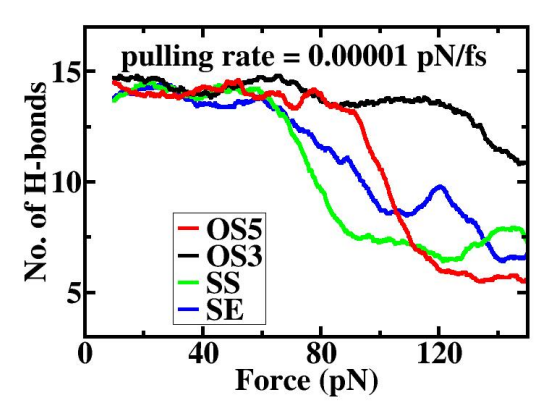

(a)

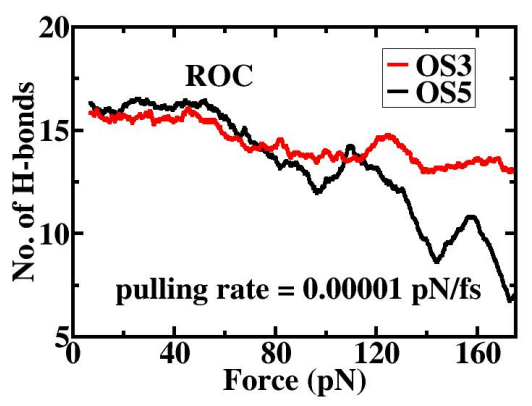

(b)

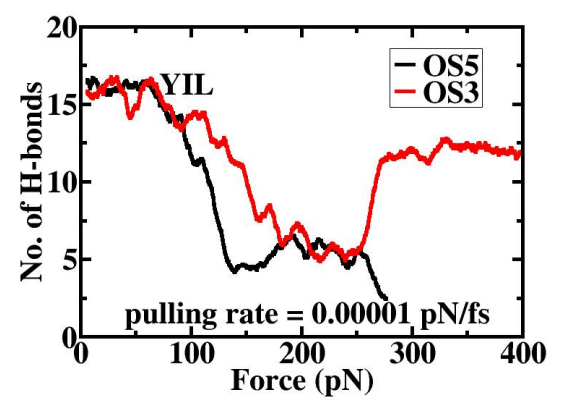

(c)

FIG. S7: Number of H-bonds vs applied force curves for different force fields at a pulling rate of $0.00001 \mathrm{pN} / \mathrm{fs}$. It is evident that for OS3 protocol, more number of H-bonds are maintained in OS3 protocol than OS5 protocol in all the force fields.

We also show the force-strain curves for both the pulling rates using three different FFs in fig. S8 and S9] In all the cases, force-strain curve of dsRNA shows jump in strain at a higher force in OS3 protocol than in OS5 protocol, regardless of the force field or pulling rate used. The magnitude of the force may vary, but qualitatively all force fields show the emergence of S-RNA when dsRNA is pulled using OS3 protocol.

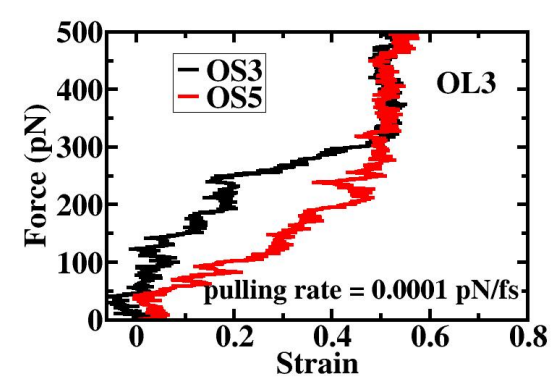

(a)

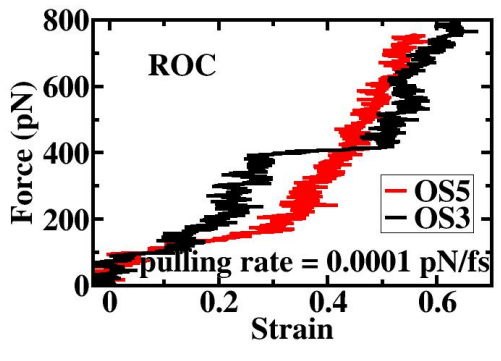

(b)



(c)

FIG. S8: Force-strain curves for different force fields at a pulling rate of $0.00001 \mathrm{pN} / \mathrm{fs}$.

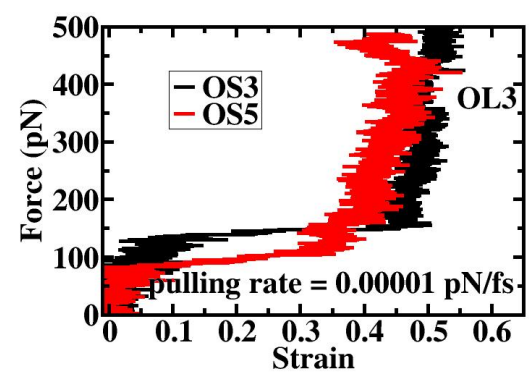

(a)

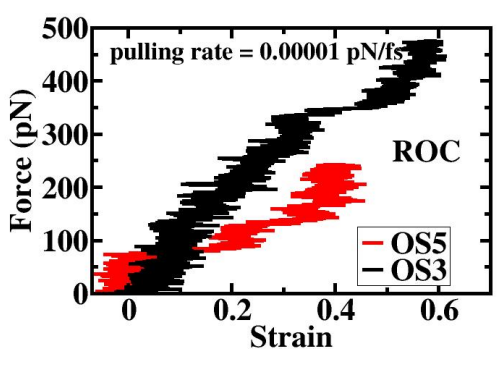

(b)

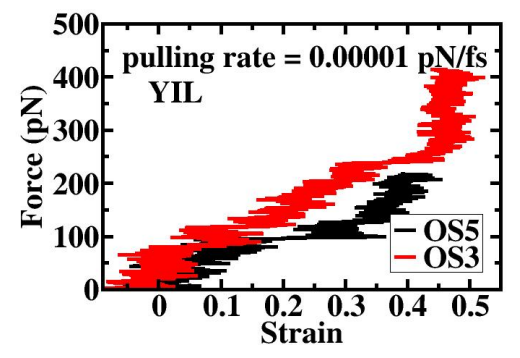

(c)

FIG. S9: Force-strain curves for different force fields at a pulling rate of $0.00001 \mathrm{pN} / \mathrm{fs}$. 
VARIATION OF STRUCTURAL PARAMETERS WHILE PULLING DS-RNA

We have calculated the average structural parameters of dsRNA using cpptraj 4 w while pulling using OS3 and OS5 pulling protocols (fig. S10). For all the parameters, it was seen that the trend of almost all parameters is different for OS3 and OS5 pulling protocols.

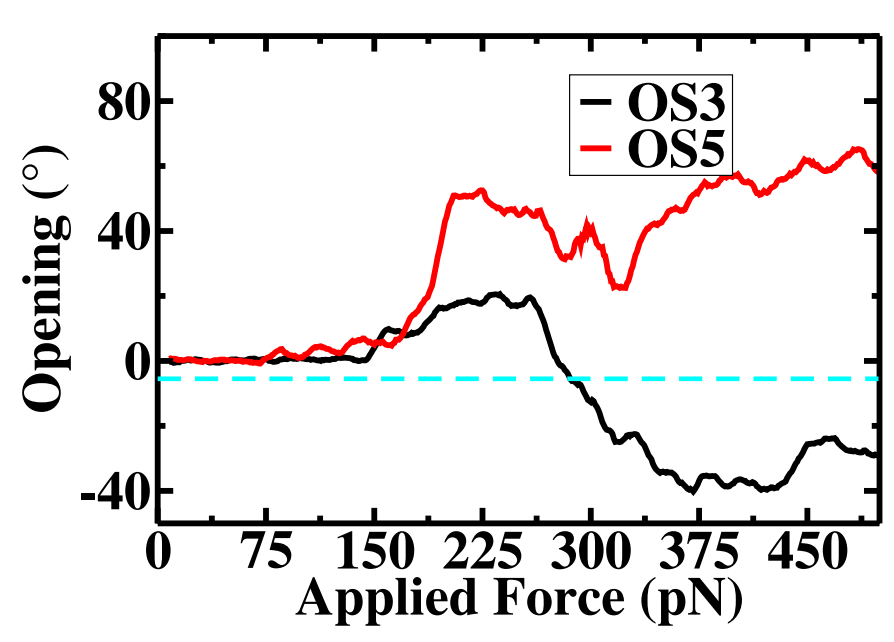

(a)

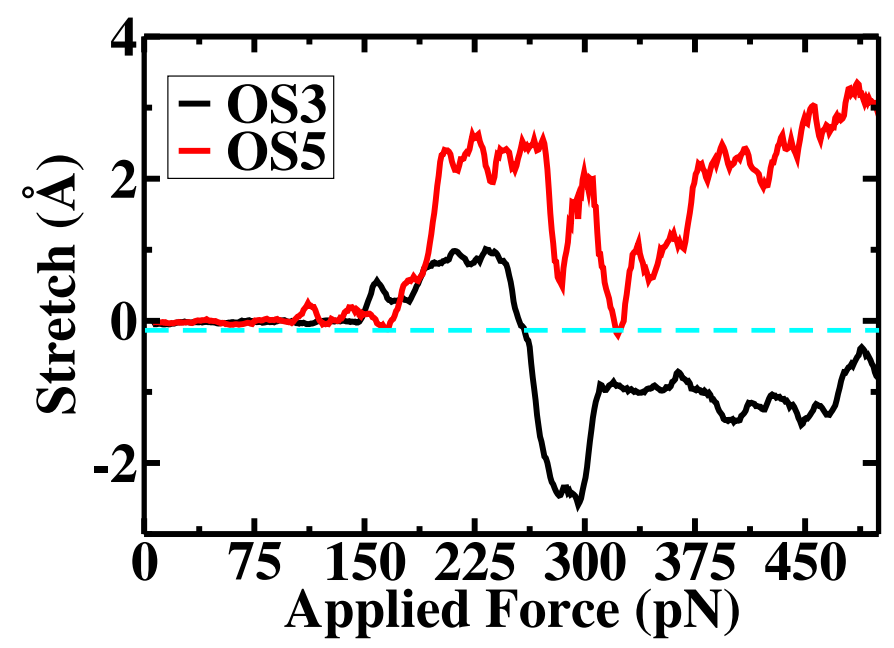

(c)

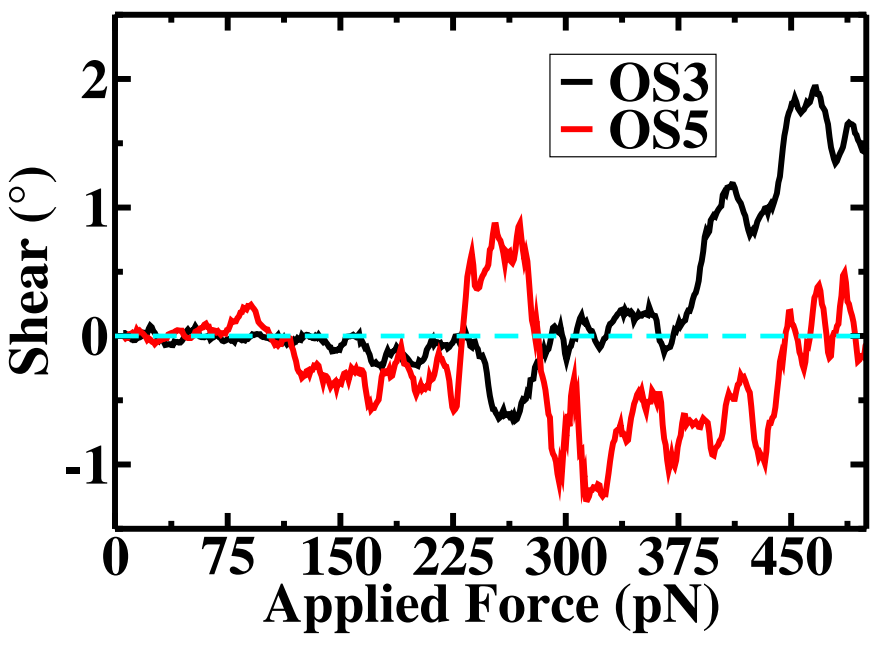

(b)



(d)

FIG. S10: Structural parameters (a) Opening, (b) Shear, (c) Stretch and (d) Slide for OS3 and OS5 pulling parameters. The blue dashed line is the average value for a crystal A-RNA structure. It is evident that the parameters for OS3 and OS5 cases vary in a very different fashion. 


\section{STRUCTURE PARAMETERS FOR S-RNA}

We present the calculated structural parameters of S-RNA. It was seen that the dsRNA was in stable S-form from $325 \mathrm{pN}$ force to $400 \mathrm{pN}$. Using cpptraj software module of AMBER 16 [4, we calculated the parameters for this force range, which are tabulated as below. To calculate the parameters of A-form of RNA, we generated a structure using NAB module of AMBER 16 software and calculated its parameters using cpptraj for comparison with S-RNA.

TABLE SI: The parameters of S-RNA and A-RNA for $d-(C G C G A A U U C G C G)$.

\begin{tabular}{lrc}
\hline \hline Parameter & S-RNA & A-RNA \\
\hline 1. Helical Twist & $8.12^{\circ}$ & $31.31^{\circ}$ \\
2. Helical Rise & $2.87 \AA$ & $2.75 \AA$ \\
3. Inclination Angle & $18.09^{\circ}$ & $16.15^{\circ}$ \\
4. Slide & $4.72 \AA$ & $-1.76 \AA$ \\
5. Buckle & $-2.20^{\circ}$ & $0.001^{\circ}$ \\
6. Propeller Angle & $11.78^{\circ}$ & $-13.73^{\circ}$ \\
7. Opening & $-33.67^{\circ}$ & $-5.49^{\circ}$ \\
8. Stagger & $-1.17 \AA$ & $0.035 \AA$ \\
9. Stretch & $-1.01 \AA$ & $-0.13 \AA$ \\
10. Shear & $0.22 \AA$ & $0 \AA$ \\
\hline \hline
\end{tabular}


* maiti@iisc.ac.in

[1] A. Pérez, I. Marchán, D. Svozil, J. Sponer, T. E. Cheatham, C. A. Laughton, and M. Orozco, Biophysical journal 92, 3817 (2007).

[2] I. Yildirim, H. A. Stern, S. D. Kennedy, J. D. Tubbs, and D. H. Turner, Journal of chemical theory and computation 6, 1520 (2010).

[3] A. H. Aytenfisu, A. Spasic, A. Grossfield, H. A. Stern, and D. H. Mathews, Journal of chemical theory and computation 13, 900 (2017).

[4] D. Case, J. Berryman, R. Betz, D. Cerutti, T. Cheatham III, T. Darden, R. Duke, T. Giese, H. Gohlke, A. Goetz, et al., University of California, San Francisco (2015). 